\title{
Essais
}

ESSAIS

Revue interdisciplinaire d'Humanités

Hors-série 6 | 2021

Agrobiodiversité et territoires

\section{Le goût d'un pays ou l'excellence gustative au service d'un territoire. La Maison Barthouil, une entreprise familiale au cœur d'un réseau local de producteurs et de pêcheurs}

Rencontre avec Jacques et Guillemette Barthouil

The taste of a country or gustatory excellence at the service of a territory. The Maison Barthouil, a family business at the heart of a local network of producers and fishermen

Meeting with Jacques and Guillemette Barthouil

\section{OpenEdition}

Journals

Édition électronique

URL : https://journals.openedition.org/essais/7966

DOI : 10.4000/essais.7966

ISSN : 2276-0970

Éditeur

École doctorale Montaigne Humanités

Édition imprimée

Date de publication : 1 mars 2021

Pagination : 191-197

ISBN : 978-2-492780-00-4

ISSN : 2417-4211

Référence électronique

" Le goût d'un pays ou l'excellence gustative au service d'un territoire. La Maison Barthouil, une entreprise familiale au cœur d'un réseau local de producteurs et de pêcheurs », Essais [En ligne], Horssérie 6 | 2021, mis en ligne le 16 mars 2021, consulté le 18 janvier 2023. URL : http:// journals.openedition.org/essais/7966 ; DOl : https://doi.org/10.4000/essais.7966 


\section{Le goût d'un pays ou l'excellence gustative au service $d^{\prime} u n$ territoire La Maison Barthouil, une entreprise familiale au coeur d'un réseau local de producteurs et de pêcheurs}

\section{Entretien avec Jacques et Guillemette Barthouil ${ }^{1}$}

La Maison Barthouil, installée à Peyrehorade (Landes), propose des foies gras et saumons fumés au goût incomparable, liés aux territoires à la rencontre desquels elle est installée : les vallées des gaves et de l'Adour, les Landes et le Pays Basque. Jacques Barthouil a dirigé l'entreprise familiale jusqu'à ces dernières années. Ces filles ont pris la suite: Guillemette est directrice de la fabrication.

Lorigine de la Maison est la charcuterie fondée en 1929 par le père de Jacques à Peyrehorade. Il y fabrique et y vend des foies gras dès ses débuts. Puis, quelque dix ans plus tard, il se met à fumer les saumons migrant dans les gaves et l'Adour.

\section{Des produits locaux}

Le canard gras, qui donne le foie gras, et le saumon de l'Adour sont deux produits issus du territoire où est implantée l'entreprise. Ils proviennent cependant de cultures différentes. Le foie gras procède de la culture du gras. Dans le Sud-Ouest de la France pour conserver la viande on faisait du confit. Du Moyen Âge au XVIII siècle, on produisait du canard gras pour conserver la viande dans la graisse. De temps en temps on trouvait un foie gras. Avec le développement de la gastronomie française aux XVIII ${ }^{e}$ et XIX ${ }^{e}$ siècles on a valorisé le foie gras en se mettant à gaver de façon systématique.

La production de saumon fumé est une idée et une pratique introduite par le père de Jacques Barthouil. C'était une idée novatrice dans le Sud-Ouest de la France où le saumon était mangé frais. On pouvait aussi le saler légèrement pour l'envoyer en barriques à Paris ou en Espagne. Constatant que le marché du saumon fumé se développait, le fondateur de la maison Barthouil part au Danemark dans les années trente pour apprendre la technique de fumage du saumon, la ramener en France et valoriser la ressource locale.

1 Dans le cadre d'un entretien mené avec Jacques Barthouil le $1^{\text {er }}$ décembre 2018 et de l'intervention de Guillemette Barthouil au séminaire, les 14 et 15 mai 2019. 
Ainsi ces produits sont travaillés de deux manières provenant de pays et de cultures fort différentes bien qu'ils soient l'un et l'autre des produits locaux. Guillemette le résume ainsi : "C'est le territoire qui a fait en sorte que l'on fabrique ces deux produits".

\section{Un choix économique : maintenir la qualité gustative}

Le choix de maintenir la production dans le registre de la qualité s'est effectué dans les années 1990, à un moment où les filières du foie et gras et du saumon fumé s'industrialisaient beaucoup. À travers le créneau de la qualité c'est le maintien de produits proposant des goûts affirmés qui est choisi. Cette orientation permet de se positionner sur un marché de niche rémunérateur et de pouvoir rester une entreprise de petite taille sans avoir besoin de développer les volumes de production, le chiffre d'affaire c'est-à-dire faire grossir l'entreprise.

L'activité de l'entreprise familiale reposait toujours dans les années 1950 sur les pêcheries de saumons de l'Adour, le marché au gras de Peyrehorade, des activités ancrées dans le territoire.

Le choix qualitatif de mettre à l'honneur le goût a amené l'entreprise à s'intéresser au domaine de la biodiversité. Ainsi l'excellence gustative s'est mise au service du territoire mais on peut aussi dire que le territoire a été mis au service de l'excellence gustative. Les produits locaux et le territoire local profitent l'un et l'autre de ce mutualisme s'il fonctionne bien.

Deux caractéristiques distinguent la qualité d'un produit : la matière première et la façon de la travailler. Il faut les maitriser complètement pour espérer obtenir un produit d'exception. Deux conceptions se confrontent sur l'importance de l'une ou de l'autre : est-ce la matière première qui prime? Est-ce la fabrication?

Dans une approche industrielle la matière première doit s'adapter au processus de fabrication. Dans les grands ateliers de fabrication industrielle, la matière première doit s'adapter au processus de production standardisé où le planning de production, les achats, la recette sont préalablement définis : la matière première doit correspondre au standard défini par la recette. Dans une approche artisanale c'est le savoir-faire qui s'adapte à la qualité de la matière première.

Ces deux approches se mêlent au sein d'une entreprise agro-alimentaire. Cependant la façon dont on organise une entreprise vouée à faire de la qualité est fondamentalement différente de celle d'une entreprise qui se consacre à faire du volume et des prix bas. 


\section{Le canard et son foie gras}

La matière première

La Maison Barthouil travaille avec cinq éleveurs-gaveurs qui sont tous installés dans un rayon de 40 kilomètres autour de l'entreprise. Ces éleveursgaveurs reçoivent le canard à un jour, l'élèvent et le gavent. Les animaux sont ensuite envoyés dans un abattoir.

Le cahier des charges que doivent respecter les éleveurs-gaveurs est propre à l'entreprise Barthouil, il n'est pas labellisé. Son respect est contrôlé par des audits internes. Parmi les critères :

- le temps d'élevage (14 semaines) est plus long que chez des éleveurs fournissant une matière première destinée à la production industrielle ;

- le maïs utilisé est produit sur la ferme ;

- $50 \%$ de l'alimentation des canards est constituée de maïs ;

- le gavage se fait au maïs entier ;

- chaque éleveur n'élève que 4200 canards par an. C'est un élevage en petit lot où 400 canards en moyenne sont élevés à la fois ;

- chaque canard dispose de 8 à $20 \mathrm{~m}^{2}$ pour s'ébattre ;

- le système de production se fait en autarcie.

Avant la venue de la grippe aviaire était utilisée une souche de canards rustiques : le Picaillon. Un canard à foie gras est un canard Mulard, c'està-dire un croisement entre un canard de Barbarie et une cane de Pékin. Ce croisement peut varier en fonction des races que l'on veut obtenir. L'entreprise Barthouil travaillait jusqu'à récemment avec le canard Mulard Picaillon.

L'épidémie de grippe aviaire l'a fait perdre. Pendant les crises sanitaires, ce sont des logiques de lutte adaptées à l'industrie agroalimentaire qui s'imposent. Ce fut le cas lors de la grippe aviaire où ont été imposés un vide sanitaire et un mode de gestion des fermes comme s'il s'agissait d'unités de production alimentaire industrielles. Ainsi, pour des questions de coût et de rationalisation de la production, l'accouveur avec qui travaillait la Maison Barthouil a arrêté de faire naître du Picaillon. Puisque les 25000 canards que lui prenait la maison Barthouil chaque année ne représentait qu'un très faible pourcentage de sa production annuelle de 2000000 de spécimens, et compte tenu des investissements qu'il avait à engager, il a décidé d'arrêter de produire du Picaillon. Du fait de cette perte, l'entreprise travaille maintenant avec une race un peu rustique mais qui reste très sélectionnée et qui donc ne convient pas tout à fait.

Depuis deux ans, en collaboration avec le Conservatoire des Races d'Aquitaine et le couvoir de la Bidouze, La Maison Barthouil travaille à retrouver des canards donnant des foies gras de qualité, à partir d'une race du bassin de l'Adour : le Kriaxera issu d'un croisement entre un canard de Barbarie et une cane locale. La filière est encore à reconstruire à partir de cette race. 
Travailler avec une souche rustique comme le Picaillon ou le Kriaxera est compliqué : il est beaucoup moins valorisable en terme de fabrication. Il faut le travailler plus longtemps. Les souches industrielles sélectionnées sont très rentables car elles sont faites pour donner de gros magrets et du foie gras. Cela signifie que travailler avec une souche rustique, moins productive, a des conséquences qu'il faut justifier pour une entreprise. Il faut pouvoir vendre les produits issus de ces souches, il faut pouvoir trouver une clientèle pour ces produits.

\section{L'organisation d'une micro-filière autour de l'excellence gustative et du respect des métiers de chacun}

Les éleveurs-gaveurs sont indépendants, c'est une caractéristique importante pour la Maison Barthouil. Certes le cahier des charges de production fait qu'ils travaillent exclusivement pour cette entreprise mais ils sont propriétaires de leur exploitation. Il s'agit qu'ils soient responsables de leur production et qu'ils gagnent de l'argent avec leur production. Cela suppose que l'entreprise Barthouil les paie bien et valorise bien la matière première qu' elle leur achète.

Cette démarche va peut-être à l'encontre d'un discours actuel où certains souhaitent que l'éleveur assure toutes les étapes de la chaîne de production : élever, transformer, vendre. Cependant, pour aboutir à un produit de qualité, mieux vaut respecter le métier de chacun et continuer à distinguer ceux qui élèvent, un métier difficile en soi, ceux qui transforment dont le savoir-faire peut être affiné durant tout une vie et ceux qui vendent. C'est un respect de chaque métier que de ne pas vouloir tout faire et de ne pas vouloir le faire à moitié.

Les industriels entendent contrôler toute la chaîne, de la production de maïs à la vente du foie gras, afin de pouvoir dégager les marges bénéficiaires qu'ils souhaitent là où ils le veulent et pour pouvoir externaliser les risques.

Cependant respecter le métier de chaque intervenant dans la chaîne de production est aussi une garantie de préservation des goûts. Un exemple : une viande achetée auprès d'un éleveur peut être d'une grand qualité mais elle peut avoir été mal préparée, mal découpée ce qui ne met pas du tout en valeur la matière première. Ainsi l'excellence gustative est mieux garantie lorsque collaborent des éleveurs et des transformateurs qui restent dans leur métier et maîtres de leur métier, de leur savoir-faire.

L'élevage d'une souche rustique demande beaucoup plus de temps, plus d'implication que celui d'une souche industrielle. Cela demande aussi plus d'attention tandis que les canards ont un comportement plus sauvage. Ainsi, les éleveurs-gaveurs qui travaillent avec l'entreprise Barthouil reviennent à un élevage qu’ont abandonné les éleveurs de souches industrielles. 
Il est important de souligner la complexité du processus qui mène de l'élevage au produit fini. Il n'est pas seulement question de sauvegarder une race, il est aussi intéressant de comprendre comment les éleveurs-gaveurs interagissent avec cette race, quelle est l'incidence économique de ce processus, comment l'entreprise Barthouil réussit à valoriser la matière première. Ce sont des canards gras entiers qui sont reçus et travaillés par l'entreprise Barthouil, choix devenu très rares dans la profession. Cela oblige à s'adapter à une matière première qui change à chaque fois et cela nécessite de valoriser tout l'animal : les carcasses sont utilisées pour faire les bouillons, la viande est cuite avec les os ce qui permet d'utiliser les os. Les cous sont également utilisés. Travailler une matière noble suppose aussi de la valoriser dans son entièreté. Cela demande également une organisation de la production à laquelle peu d'entreprise aujourd'hui sont capables de s'ajuster puisqu'il faut s'adapter en permanence à la matière première. On ne peut pas suivre un planning reconduit chaque semaine.

$\mathrm{Au}$ sein du processus de fabrication, la Maison Barthouil est l'une des dernières à pratiquer l'éviscération à froid. Cela signifie que le foie gras est laissé à mâturer à l'intérieur de la carcasse du canard : comme pour la maturation de viande, un processus enzymatique va développer les goûts du foie gras. Seuls du sel et du poivre sont ajoutés au foie car l'objectif du processus de fabrication est bien de faire ressortir le goût de la matière première.

\section{Le saumon}

Si la Maison Barthouil continue à travailler les saumons de l'Adour elle est aussi spécialisée dans le travail de tous les saumons sauvages de l'Atlantique, ainsi que du saumon label rouge norvégien et du saumon bio d'Écosse. Néanmoins le saumon de l'Adour est un des produits fabriqué dès les origines de l'entreprise, un de ses produits emblématiques.

Le saumon de l'Adour a un cycle de vie et un cycle migratoire très intéressants. Après être né et avoir passé quelques années en rivière, il migre dans l'océan, va grandir près du Labrador avant de revenir dans la rivière dans laquelle il est né. Le saumon de l'Adour n'est autorisé à être pêché qu'en rivière. Avant 1939 il constituait une ressource halieutique très abondante. Les barrages installés sur les rivières ont perturbé la ressource en empêchant la remontée des saumons dans les frayères de montagne.

Aujourd'hui la gestion de la ressource est au cœur d'une lutte entre pêcheurs professionnels et pêcheurs amateurs, à la ligne, les uns accusant les autres de prélever de la ressource. En terme de développement du territoire il s'agit d'une confrontation intéressante de deux conceptions d'un rapport à la nature et aux territoires. Souhaite-t-on faire vivre nos territoires seulement par le tourisme et les loisirs? Ou une cohabitation est-elle possible avec un monde économique qui respecte ces ressources? 
Pour les pêcheurs à la mouche, amateurs, les pêcheurs professionnels vivent d'une ressource qu'ils prélèvent au détriment de leur activité de pêche à la ligne qui pourrait créer du tourisme et de l'emploi. Ils se confrontent à des pêcheurs professionnels qui, tout en essayant de vivre de leur métier, sont engagés depuis 15 ans dans un programme en collaboration avec l'INRA et l'université de Pau pour comprendre d'où viennent les saumons et comment il retrouvent la rivière où ils sont nés. De plus un COGEPOMI (Comité de Gestion des Poissons Migrateurs) a été mis en place pour gérer la ressource et depuis dix ans la population de saumon sauvage est en augmentation. Le combat entre pêcheurs amateurs et pêcheurs professionnels est vain et même pathétique alors que les deux parties auraient intérêt à s'entendre pour batailler contre les barrages qui forment le véritable problème de gestion de la ressource en saumon.

\section{Organisation de la production}

L'entreprise Barthouil est en contact avec des pêcheurs locaux de saumon. Puisque le poisson pêché est sauvage, aucun contrôle n'est possible en matière de régularité du volume de matière première fournie : certaines semaines verront arriver dans l'entreprise beaucoup de saumons et d'autres semaines beaucoup moins. La Maison Barthouil ayant fait le choix de ne travailler que du saumon frais, elle doit adapter l'organisation de sa production à cet approvisionnement irrégulier qui induit une irrégularité du volume de travail en fonction du moment : certaines semaines on travaillera beaucoup de matière première et d'autres presque pas. Cela suppose d'avoir des équipes solides et motivées et d'assurer la gestion humaine et le management nécessaire pour cela. Cette organisation contraignante résultant du choix de ne travailler que du saumon frais s'explique par la volonté d'obtenir un produit de meilleure qualité.

\section{Le processus de fabrication}

Les saumons sont reçus entiers. Ils sont scarifiés, filetés à la main, salés au sel de Salies-de-Béarn. Ils sont séchés et fumés dans des fumoirs danois selon les mêmes méthodes que dans les années cinquante. Ils sont enfin tranchés à la main puis vendus.

\section{Le produit et sa relation gustative au territoire}

Tous les saumons sont transformés de la même manière qu'ils soient sauvages, d'élevage ou bio. Il s'agit de saumons de la même espèce, le Salmo salar mais elle prend des goûts, des textures et des couleurs complétement 
différents selon ce dont elle s'est nourrie et le lieu où elle a grandi. Cela illustre de façon remarquable le lien entre biodiversité gustative et relation au territoire. Les dégustations comparatives de saumons sont rares mais mettent en évidence ce rapport.

Les relations avec les éleveurs, les pêcheurs et les clients rendent l'entreprise vivante. Oui la Maison Barthouil existe pour réaliser des bénéfices. Ces bénéfices lui permettent, par l'indépendance qu'ils lui accordent, de faire des choix d'importance. Mais elle existe également en relation à un territoire, elle fait partie d'un territoire. C'est au moins aussi important que de faire du bénéfice. Cette raison a été déterminante pour que Guillemette Barthouil vienne travailler au sein l'entreprise familiale.

Le travail quotidien de l'entreprise est de transformer une matière première de grande qualité, la mettre en valeur en faisant ressortir ses caractéristiques territoriales et de satisfaire des clients qui recherche le goût d'un pays. Il s'agit d'un équilibre difficile à trouver qui se base sur des relations humaines, un attachement viscéral au territoire et bien sûr une certaine indépendance financière. Une quête qui, aujourd'hui, peut motiver beaucoup de jeunes professionnels à faire vivre nos territoires et donner du sens à nos métiers, à l'image de Guillemette Barthouil. 